\title{
Striking While the Iron is Hot: Understanding the Biological and Neurodevelopmental Effects of Iron Deficiency to Optimize Intervention in Early Childhood
}

\author{
Jenalee R. Doom • Michael K. Georgieff
}

Published online: 23 August 2014

(c) Springer Science + Business Media New York 2014

\begin{abstract}
Prenatal and early postnatal iron deficiency (ID) is associated with long-term neurobiological alterations and disruptions in cognitive, social, and behavioral development. Early life ID is particularly detrimental as this is a period of rapid neurodevelopment. Even after iron supplementation, cognitive and social disruptions often persist in formerly iron-deficient individuals. Observational studies of the acute and long-term effects of early life ID yield different results based on the timing of ID. Further, intervention studies demonstrate some improvement for certain domains but still show residual effects years later, which are dependent on the timing of ID and treatment. This review will cover the effects of ID during infancy and early childhood on brain structure and function, cognition, and behavior in relation to preclinical models of ID and sensitive periods of human brain development.
\end{abstract}

Keywords Iron deficiency - Neurodevelopment · Infancy $\cdot$ Toddlerhood $\cdot$ Nutritional interventions

J. R. Doom ( $₫) \cdot$ M. K. Georgieff

Institute of Child Development, University of Minnesota,

Minneapolis, MN, USA

e-mail: doomx008@umn.edu

M. K. Georgieff

e-mail: georg001@umn.edu

J. R. Doom · M. K. Georgieff

Center for Neurobehavioral Development, University of

Minnesota, 51 E River Rd., Minneapolis, MN 55413, USA

M. K. Georgieff

Department of Pediatrics, University of Minnesota, Pediatric Neonatology, 6th Floor East Building, MB630, 2450 Riverside Ave, Minneapolis, MN 55454, USA

\section{Introduction}

Iron deficiency (ID) in prenatal and early postnatal life produces long-term alterations in neurobiological processes as well as disruptions in cognitive, social, and behavioral development. ID is particularly detrimental if it occurs during the fetal period or the first few years of life while neural systems are rapidly developing. Even after iron treatment, cognitive abilities in individuals who were iron deficient early in life are rarely fully restored. This review will cover the effects of ID on neural structure and function, cognition, and behavior during infancy and early childhood.

This review is grounded on 5 core principles of nutrients and brain development, which are especially applicable to ID. (1) The brain is not homogeneous; thus, it should not be viewed as a single organ. The brain consists of different types of cells (e.g., neurons and glial cells) and distinct regions (e.g., striatum, hippocampus, prefrontal cortex, cerebellum) that do not operate in the same way nor respond similarly to environmental factors. Any research on the neurobehavioral effects of early ID must specifically relate to the cell types and brain region hypothesized to be affected. (2) The separate regions of the brain have different developmental trajectories. As a result, it is likely that the greatest effects of ID will occur in brain regions that are rapidly developing and whether that development is specifically dependent on iron. It should be noted that regions with strong anatomical connections to these rapidly developing regions may also be at risk, despite having a later developmental trajectory. This occurs because brain regions typically work together in circuits to optimize behavioral functioning. Thus, insults to one brain region may negatively affect development in other later-developing regions. (3) Timing is critical to understand 
neurobehavioral abnormalities resulting from ID. Outcomes vary depending on the timing of the insult as well as what is developing in specific regions of the developing brain during the time of the insult. For example, ID during the late prenatal period leads to delayed neural processing speed [1] and deficits in recognition memory that can be detected through event-related potentials (ERP) [2]. The recognition memory deficits are consistent with altered development of the hippocampus, which is predominant during the late fetal and early postnatal period. In contrast, ID acquired between 6 and 36 months postnatally is typically not associated with memory deficits, but rather with abnormalities in affect and motor skills. This later phenotype is consistent with the development of systems governing monoamine transmission in the frontal cortex and mid-brain, respectively, which occurs primarily between birth and 3 years of age. Consequently, a "signature effect" in which a micronutrient deficiency generates one specific neurobehavioral phenotype is not likely. (4) Micronutrient deficiencies rarely occur in isolation. In most malnourished populations, it is likely that several cooccurring nutrient deficiencies that affect brain development are also present (e.g., Vitamin A, Vitamin B12, Vitamin D, iodine, selenium, copper, folate, choline, zinc), and thus it is difficult to determine which nutrient is responsible for particular effects. (5) Correct assessments should be used in order to accurately measure the processes and outcomes of interest. The child's developmental stage must also be taken into account in order to choose assessments that are appropriate for the child and sensitive enough to test the effects of ID. Unfortunately, there are currently no assessments that are both sensitive and specific enough to detect ID through neurodevelopmental measures alone without supplemental serum iron measures. However, it is possible to use tests that assess brain regions that may have been the most affected by ID. For example, if a hippocampal insult is expected following early life ID, a neurocognitive test of hippocampal function will provide more valuable information than a general cognitive test (i.e., the Bayley Scales of Infant Development) which may not be sensitive enough to detect effects on specific neural systems. Likewise, a test specific to prefrontal cortex function will likely be more appropriate for later postnatal ID.

\section{Iron Biology}

The developing brain is a highly metabolic organ, consuming $50 \%$ of the energy utilized by the baby [3]. All highly metabolic, rapidly developing organs have high iron requirements because iron supports fundamental metabolic processes (e.g., ATP generation through its role in cytochromes). As will be discussed below, the risk of ID in the developing human is not equal across all pediatric time epochs. The late fetal/early neonatal period and late infancy/toddler period are times when children are more likely to be iron deficient. The neurobehavioral effects of deficiencies in those time periods are a function of which iron-dependent processes are most active. Three fundamental iron-dependent systems are rapidly developing from mid-late gestation through the first 3 postnatal years: monoamine (e.g., dopamine) neurotransmission, myelination, and the hippocampus. Thus, it is not surprising that behavioral studies of children who are iron deficient during that time period show that they have abnormalities in mood/affect, speed of processing, learning, and memory, respectively [2, 4-6]. Other iron-sensitive systems developing during that time include the striatum and early prefrontal cortex as evidenced by abnormalities in attention and other high-end processing following iron deficiencies in toddlerhood $[6,7 \cdot, 8]$. The iron-dependent biology behind these effects has been well worked out using multidisciplinary approaches from pre-clinical models through human investigation.

The effects on the monoamine system have been known for 40 years [9]. The enzymes that synthesize dopamine and serotonin, tyrosine and tryptophan hydroxylase, respectively, contain iron and their enzymatic activity is dependent on the iron state. Rodent models of prenatal and postnatal dietary iron deficiency show significant changes not only in dopamine synthesis, but also in expression of dopamine D1 and D2 receptor classes and dopamine and serotonin re-uptake mechanisms. These abnormalities are present during the period of iron deficiency but also in the adult animal long after repletion [10], suggesting long-term or permanent reprogramming of the monoamine system. Human studies (see below) corroborate these findings in the neonate [11] through young adulthood [8] in that they demonstrate short and long-term changes in dopamine dependent affect, frontal lobe function and motor activity.

The effects on the fatty acid profile and myelin content of the brain have also been thoroughly investigated. Iron is essential to the enzymatic activity of desaturases that determine the fatty acid profile of myelin [12, 13]. ID causes significant reductions in myelin content and alters the fatty acid profile of that myelin. In addition, oligodendrocytes that synthesize myelin are highly metabolic cells with high iron requirements. Oligodendrocyte expression of myelin basic protein and PLP-1 \& -2 is compromised by ID and the effects last beyond the period of ID [14]. Physiologically, these changes to the myelin system likely contribute to the reduced speed of processing observed in neonates [1], infants [15] and toddlers [4] with current or previous ID.

Neuronal differentiation is a highly metabolic process that requires an adequate and steady source of iron. The efficacy of a neuron is dependent in great part on the 
complexity of its structure. In the developing hippocampus, this translates into the complexity of the apical dendritic arbor, particularly in area CA-1. This area is where genes that regulate synaptic plasticity are particularly active in changing the structure of the region (e.g., elongation, branching, synaptic head maturation) in response to experience. Thus, the hippocampus is particularly important in fundamental recognition learning and memory of objects and orientation in space. Greater arbor complexity and hippocampal size is associated with better learning and memory performance. Hippocampal dendritic arbor complexity, in turn, is largely dependent on two factors: cellular energy metabolism and glutamatergic neurotransmission. Both are compromised by early life ID [16-18] with resultant structural abnormalities [19, 20•], reduced electrophysiologic potential [21] and compromised learning and memory $[19,22]$. The effect is due to iron and not anemia as evidenced by these changes being present in non-anemic models of ID where only hippocampal neuronal iron has been reduced through genetic techniques [19, 20•]. There is a critical time period for iron delivery to the hippocampus, after which provision of iron will not rescue the phenotype (see below) [20 $]$.

A more recent set of investigations show that ID alters brain gene expression both during the period of ID and into adulthood. These short- and long-term gene changes are numerous (i.e., over 300 genes) and are found throughout the brain [14], specifically in the hippocampus [23] and are particularly salient to the system abnormalities discussed above; i.e., monoaminergic and glutamatergic neurotransmission [10, 24]; myelination [14] and hippocampal synaptic plasticity [19, 20•, 23].

Based on the biology described above, it should not be surprising that the iron deficient brain does not function properly and results in abnormal behavior patterns during the period of iron deficiency. However, the more important public health issue revolves around the mechanisms of the long-term effects of early life ID that persist in spite of iron treatment. Unlike the hematologic effects (e.g., anemia, microcytosis) that appear to revert to normal with iron therapy, multiple pre-clinical and clinical studies demonstrate that the brain remains abnormal well into the future. The cost to society of this effect in humans in terms of lost intellectual potential (estimated to 10 IQ points), lost job potential and increased risk of adult psychopathologies including depression, anxiety and schizophrenia $[8,25]$ is the more concerning consequence of early life ID.

Investigations in pre-clinical models provide mechanistic insight into these long-term effects [10, 14, 23, 24, 26, 27•]. The studies center on two, not mutually exclusive, potential mechanisms. The first revolves around the concept of critical periods of brain development [28]. Fundamentally, this theory states that if the structure is built wrong during a critical period of development, it remains permanently abnormal. Since the 1950's studies have demonstrated that certain brain areas require proper stimulation at a specific point in development to ensure normal structure and function. The classic work that Hubel and Weisel performed (for which they received the Nobel Prize) demonstrated the importance of appropriately timed visual input to properly form ocular dominance columns in the occipital cortex [29]. Hensch and colleagues [28] have been instrumental in identifying the cellular markers of the opening and closing of critical periods. The opening of the critical period is heralded by the appearance of parvalbumin positive GABA interneurons, while the closing of the period occurs when these interneurons surround the neurons with perineuronal nets made of chondroitin sulfate. Early life iron deficiency delays the opening of the critical period in the hippocampus, but also closes it prematurely, thereby reducing the window of opportunity to have normal development with treatment [27•]. The timing of the critical period is during the period of rapid dendritogenesis on the hippocampus and the restoration of iron to an iron deficient hippocampus within the critical period rescues it at the gene, structural and behavioral levels [20•]. Conversely, late treatment results in abnormalities at all of the levels in adulthood [20•].

The second concept is the developmental origins of adult health and disease (DoHAD) hypothesis [30]. This concept is driven by environmental epigenetic modification of chromatin during early life that results in life-long abnormal gene processing. Brain derived neurotrophic factor (BDNF) is an important synaptic plasticity growth factor that is suppressed by early life ID in the rodent [26, 31•]. Its expression remains suppressed in adulthood in spite of iron repletion. BDNF is an epigenetically modifiable gene [32], and the possibility exists that early iron deficiency can alter its expression through epigenetic mechanisms. This area deserves further exploration given the large number of brain genes affected by early life ID.

Ultimately, these abnormalities in biochemistry, metabolism, structure, and neurophysiology translate into abnormal behaviors in the preclinical models. Formerly iron deficient adult rodents exhibit abnormalities in learning and memory [22], social-affective behavior [10, 33], paired-pulse inhibition (a model of schizophrenia) [21], and frontal lobe function [34]. These abnormalities map directly onto the types of behaviors that remain abnormal in humans following early life ID (see below).

\section{Human Studies: Linking Pre-clinical to Clinical} Investigations

Overall, ID in infancy and early childhood has been linked to lower IQ and school achievement, poorer attention and memory, slower language development, deficits in motor 
skills, and disruptions in socioemotional development [7•, 35]. The effects of ID are most detrimental during the prenatal and early postnatal periods (before 3 years) when rapid myelination is occurring. Myelination deficits may slow the speed of processing or decrease synaptic efficiency. In pre-clinical models, ID decreases expression of serotonin [36] and BDNF [26]. Future research should explore interactions between neurotransmitter systems following early life ID because alterations in expression of several neurotransmitters (e.g., serotonin, dopamine, glutamate) have consequences for psychopathology, including anxiety, depression, schizophrenia, and autism. Anxiety, depression and schizophrenia have all been associated with early life ID [8, 25]. The following sections review recent research on the effects of early ID, broken down by the timing of ID (prenatal vs. postnatal) and type of study (intervention vs. observation).

\section{Observational Studies of Fetal/Neonatal ID}

The acute effects of ID in the term or preterm newborn include poorer discrimination memory [2], altered temperament and interactions with the caregiver [11], slower speed of neural processing [1] and a greater number of abnormal neurological reflexes [37]. The risk of neonatal ID to long-term neurobehavioral function was initially reported in a study of children in the lowest quartile of cord serum ferritin at birth who demonstrated significantly lower IQ and poorer language ability, tractability, and fine motor skills at 5 years [38]. One-year-old children who were born to diabetic mothers and had lower cord serum ferritin concentrations at birth showed poorer explicit memory as measured by recalling a series of events after a delay [39]. While diabetes mellitus during pregnancy confers multiple risks to fetal neurodevelopment besides iron deficiency (e.g., hypoglycemia and hypoxia), these factors were controlled for in this series of studies [40]. In 3.5-year-olds whose neonatal ID had resolved, those with lower serum ferritin concentrations at birth had poorer immediate, delayed, and working learning and memory [41]. Indeed, the poorness of their performance was directly related to their degree of neonatal ID. Interestingly, providing children with strategies for the memory tasks partially alleviated problems, suggesting that alternative learning strategies may help children with lower iron levels at birth [41]. Finally, offspring of women who had iron deficiency anemia with a hemoglobin concentration $<100 \mathrm{~g} / \mathrm{L}$ during pregnancy have a 3.7 fold greater risk of schizophrenia as adults. The increase in risk was directly related to the degree of maternal ID during pregnancy [25].

\section{Observation Studies of Postnatal ID}

The acute and chronic effects of postnatal ID have been well-chronicled in a series of more than 40 observational studies over the past $30+$ years [35]. Acute ID resulting in slower neural conduction velocities have been reported in 6-month-olds with ID [15], which has been attributed to disruptions in myelination. Toddlers with ID have a lower developmental quotient than iron sufficient toddlers, and the gap in cognitive functioning between these groups widens with age [6]. Toddlers currently suffering from ID also have socioemotional alterations, including increased hesitancy and wariness in novel situations [5]. These are thought to relate to alterations in monoamine neurotransmission [35]. Infants with ID anemia demonstrate poorer performance for both gross and fine motor skills at 9 months of age [42] and abnormal sleep patterns [43].

Overall, the cognitive, motor, and socioemotional effects of acute ID in infancy carry forward long-term, persisting into adolescence and adulthood long after ID has resolved [35, 44••]. Early postnatal ID negatively impacts visual processing and myelination, as 4-year-old, formerly iron deficient children have longer visual evoked potential latencies than controls who were never anemic [4]. Abnormal sleep patterns [43], an increased risk of anxiety and depression [8], and poorer inhibitory control [45] have been described in formerly ID children, adolescents and adults.

\section{Intervention Studies}

The results of iron intervention studies in infants are mixed, mostly as a function of the differences in intervention strategies. In general, two types of iron interventions have been studied: (1) treatment of children with current ID, and (2) prevention supplementation in children who are at risk for ID. Treatment studies are consistently positive in their results, either hematologically, neurodevelopmentally, or both. There are more mixed results in the prevention studies. This is likely due to the uncertainty of ID in the at-risk population (whether iron sufficient or extremely deficient) or the presence of multiple micronutrient deficiencies, which makes it difficult to isolate the impact of iron. Comparisons in treated versus untreated children (or children's pre-treatment state) demonstrate that treatment is better than no treatment. Treated children versus always iron sufficient controls show residual effects, which suggests that treatment does not resolve all effects. This latter effect may vary by age at ID detection. 


\section{Prenatal Intervention Studies}

Prenatal iron and folic acid supplementation in pregnant women from rural Nepal who had a high risk of ID resulted in positive effects on neurodevelopmental outcomes, including inhibitory control, working memory, and motor functioning, at ages 7-9 years compared to the control group [46]. In this same study, supplements between ages 12-36 months did not provide additional benefits [47]. As a result, the timing of supplementation is critical, and it appears that earlier (even during the prenatal period) is better for the developing brain [48, 49••]. Another supplementation study during pregnancy found that iron deficient mothers who began iron supplementation before 20 weeks gestation had infants with higher birth weights, a smaller likelihood of low birth weight (LBW) infants, and fewer preterm LBW infants compared to women who started supplementation if iron deficient at 28 or 38 weeks [50]. This study suggests that iron treatment may improve conditions related to poorer cognitive outcomes, including LBW status.

\section{Postnatal Intervention Studies}

Several postnatal intervention studies report marked improvements in cognitive and motor skills. The majority of studies report neurological improvements even after the period of iron treatment, suggesting a sensitive or critical period for certain iron-dependent processes in the developing brain [35]. Most studies of iron supplementation in infants with ID show positive effects in the treatment group [51], and many studies of ID prevention in infancy also demonstrate positive neurological and behavior effects in addition to improved iron status [52, 53]. In iron supplementation in children under 2 years of age, those who were anemic in infancy often fail to catch up with non-anemic infants [54]. In one study, 5-year-olds who received iron supplementation in infancy to correct ID anemia still showed lower physical activity levels, less positive affect, and less verbalization [55], again emphasizing the longterm risk when ID occurs early in life. One study showed no effect of iron supplementation, which was attributed to later timing of supplementation (12-36 months) that could have missed an early period of rapid brain development [56].

Preterm and LBW infants are at higher risk for postnatal ID because iron is accreted in the last trimester. In a study of LBW infants born between 2,000 and 2,500 $\mathrm{g}$ who received 0,1 , or $2 \mathrm{mg} / \mathrm{kg} /$ day of iron supplements between 6 weeks and 6 months of age, treatment efficacy differed by neurodevelopmental outcome [57•]. At 3.5 years, although there was no effect of the intervention for IQ,
$12.7 \%$ of the LBW placebo (i.e., $0 \mathrm{mg} / \mathrm{kg} / \mathrm{d}$ ) group had scores on the Child Behavior Checklist above the clinical cutoff $(\mathrm{T}>63$; $>90$ th percentile) versus $2.9,2.7$, and $3.2 \%$ for the $1,2 \mathrm{mg}$, and normal birth weight groups, respectively. Consequently, iron supplementation in LBW infants during the first 6 months of life may reduce clinical symptoms in early childhood. Another study of VLBW infants $(<1,301 \mathrm{~g})$ randomized infants into early (mean $=14$ postnatal days) or late iron supplementation (mean $=61$ postnatal days), and the results indicated a trend toward better neurocognitive and motor development for the group with early versus late iron supplementation [58].

In general, neurological domains that are affected by acute ID in toddlerhood show persistent negative effects over time even after correction of ID [7•,35]. Cognitive abilities such as math and writing are often poorer in formerly iron deficient children, which is consistent with abnormalities in hippocampal and prefrontal cortical development [8].

\section{Conclusions and Potential Confounders in Attributing Outcomes to ID}

Is there evidence of a specific neurodevelopmental biomarker for ID? Although there are multiple behavioral sequelae of ID, it is not likely that any are specific to ID. Traditionally recognized biological abnormalities of early life ID are on monoamine neurotransmission, myelination, and neuronal growth/differentiation with their attendant effects on behavior, mood/affect alterations, reduced speed of processing, and poorer learning and memory. However, one or multiple other nutrient deficiencies can cause abnormalities in each of those domains. For example, manganese toxicity and iodine deficiency can cause monoamine abnormalities. Hypoxia, and iodine and LCPUFA deficiencies can cause hypomyelination. Zinc, oxygen and protein deficiency can cause truncated dendritic trees and abnormal synaptogenesis. Thus, while it would be attractive to have a "signature" effect of early life ID on the brain, this is not likely. Nutrient deficiencies often occur simultaneously, which makes it difficult to determine the effects of specific micronutrient deficiencies on neurodevelopment.

Similarly, non-nutritional risk factors for the brain also co-occur with ID. Internationally adopted children who spent part of their lives in institutional care are an example of a group that has a high prevalence of ID (approximately $26 \%)$ recognized at the time of adoption [59]. However, many of the correlates of ID that are present in internationally adopted children are also residual symptoms of early institutional care, which usually involves some degree 
of stimulus and/or social deprivation. For example, the duration of institutional care and severity of ID are independent predictors of both IQ and executive function skills 12 months post-adoption [60]. Additionally, the phenotype of ID varies as a result of the timing of the deficiency, so a consistent marker across time is elusive. As a result, it is probable that other factors affecting development may produce a similar phenotype to ID, but there are likely no neurobiological or behavioral markers specific to ID.

Another possible explanation for observed cognitive and social effects with ID includes functional isolation [11]. As discussed above, children with ID are often hesitant and wary and engage less with their caregivers [11, 61]. Caregivers may view the children as less problematic and give them less attention. As a result, children receive less stimulation from caregivers and the environment, which slows brain development.

Research on the effects of ID on neurodevelopment, cognition, and behavior in infancy and early childhood suggests long-term disruptions in functioning even after restoring normal iron levels. Many of these effects are consistent with enduring striatal-nigral and striatal-frontal dopaminergic circuitry alterations. Interestingly, frontal lobe-mediated functions including attention, planning, and inhibitory control are also affected following early ID [6, 8], which would not be expected as the frontal lobe is not experiencing rapid development during very early postnatal life. It is likely that these outcomes are developmental side effects of rapidly developing brain regions such as the striatum and hippocampus, including suboptimal connections between the frontal lobe and these primary regions. As a result, considering the effects of early ID in relation to timing (neurodevelopmental stage), severity, and duration is essential to understanding outcomes in the heterogeneous brains of infants and young children.

Acknowledgments We are grateful to have support from the following Grants: R01 HD-29421-18, P01 HD039386, 2 RO1 NS3445810 , and T32MH015755.

Disclosure Jenalee R. Doom and Michael K. Georgieff declare that they have no conflict of interest.

Human and Animal Rights and Informed Consent This article does not contain any studies with human or animal subjects performed by any of the authors.

\section{References}

Papers of particular interest, published recently, have been highlighted as:

- Of importance

-• Of major importance
1. Amin SB, Orlando M, Eddins A, MacDonald M, Monczynski C, Wang H. In utero iron status and auditory neural maturation in premature infants as evaluated by auditory brainstem response. J Pediatr. 2010;156(3):377-81.

2. Siddappa AM, Georgieff MK, Wewerka S, Worca C, Nelson CA, deRegnier R-A. Auditory recognition memory in iron-deficient infants of diabetic mothers. Pediatr Res. 2004;55:1034-41.

3. Kuzawa CW. Beyond feast-famine: brain evolution, human life history, and the metabolic syndrome. In: Muehlenbein M, editor. Human evolutionary biology. Cambridge: Cambridge University Press; 2010. p. 518-27.

4. Algarín C, Peirano P, Garrido M, Pizarro F, Lozoff B. Iron deficiency anemia in infancy: long-lasting effects on auditory and visual system functioning. Pediatr Res. 2003;53(2):217-23.

5. Lozoff B, Klein NK, Nelson EC, McClish DK, Manuel M, Chacon ME. Behavior of infants with iron deficiency anemia. Child Dev. 1998;69:24-36.

6. Lozoff B, Jimenez E, Hagen J, Mollen E, Wolf AW. Poorer behavioral and developmental outcome more than 10 years after treatment for iron deficiency in infancy. Pediatrics. 2000; 105:E51.

7. - Georgieff MK. Long-term brain and behavioral consequences of early iron deficiency. Nutr Rev. 2011; 69:S43-8. This paper is a review of the long-term effects of pre-and post-natal ID in both humans and in model systems. Alterations in neurotransmitter systems and changes in gene expression are discussed.

8. Lukowski AF, Koss M, Burden MJ, et al. Iron deficiency in infancy and neurocognitive functioning at 19 years: evidence of long-term deficits in executive function and recognition memory. Neuroscience. 2010;13(2):54-70.

9. Youdim MBH, Green AR, Bloomfield MR, Mitchell BD, Heal DJ, Grahamesmith DG. The effects of iron deficiency on brain biogenic monoamine biochemistry and function in rats. Neuropharmacology. 1980;19:259-67.

10. Unger EL, Hurst AR, Georgieff MK, et al. Behavior and monoamine deficits in pre- and peri-natal iron deficiency are not corrected by early postnatal moderate or high iron diet in rats. J Nutr. 2012;142:2040-9.

11. Wachs TD, Pollitt E, Cueto S, Jacoby E, Creed-Kanashiro H. Relation of neonatal iron status to individual variability in neonatal temperament. Dev Psychobiol. 2005;46(2):141-53.

12. Connor JR, Menzies SL. Relationship of iron to oligodendrocytes and myelination. Glia. 1996;17(2):83-93.

13. Ortiz E, Pasquini JM, Thompson K, et al. Effect of manipulation of iron storage, transport, or availability on myelin composition and brain iron content in three different animal models. J Neurosci Res. 2004;2004(77):681-9.

14. Clardy SL, Wang X, Zhao W, et al. Acute and chronic effects of developmental iron deficiency on mRNA expression patterns in the brain. J Neural Transm Suppl. 2006;71:173-96.

15. Roncagliolo M, Garrido M, Walter T, Peirano P, Lozoff B. Evidence of altered central nervous system development in infants with iron deficiency anemia at 6 mo: delayed maturation of auditory brainstem responses. Am $\mathrm{J}$ Clin Nutr. 1998;68(3):683-90.

16. Rao R, Tkac I, Townsend EL, Gruetter R, Georgieff MK. Perinatal iron deficiency alters the neurochemical profile of the developing rat hippocampus. J Nutr. 2003;133:3215-21.

17. De Ungria $M$, Rao R, Wobken JD, Luciana $M$, Nelson CA, Georgieff MK. Perinatal iron deficiency decreases cytochrome c oxidase activity in selective regions of neonatal rat brain. Pediatr Res. 2000;48:169-76.

18. Dallman PR. Biochemical basis for the manifestations of iron deficiency. Annu Rev Nutr. 1986;6:13-40. 
19. Carlson ES, Tkac I, Magid R, et al. Iron is essential for neuron development and memory function in mouse hippocampus. J Nutr. 2009;139(4):672-9.

20. - Fretham SJB, Carlson ES, Wobken J, Tran PV, Petryk A, Georgieff MK. Temporal manipulation of transferrin receptor-1 dependent iron uptake identifies a sensitive period in mouse hippocampal neurodevelopment. Hippocampus. 2012;22:16911702. This article demonstrated that the spatial memory abnormalities seen with ID are due to neuronal iron deficiency and not to anemia and that there is a specific critical period for iron in the developing hippocampus.

21. Pisansky MT, Wickham RJ, Su J, et al. Iron deficiency with or without anemia impairs prepulse inhibition of the startle reflex. Hippocampus. 2013;23(10):952-62.

22. Schmidt AT, Waldow KJ, Grove WM, Salinas JA, Georgieff MK. Dissociating the long-term effects of fetal/neonatal iron deficiency on three types of learning in the rat. Behav Neurosci. 2007;2007(121):475-82.

23. Carlson ES, Stead JDH, Neal CR, Petryk A, Georgieff MK. Perinatal iron deficiency results in altered developmental expression of genes mediating energy metabolism and neuronal morphogenesis in hippocampus. Hippocampus. 2007;17:679-91.

24. Tran PV, Dakoji S, Reise K, Storey K, Georgieff MK. Fetal iron deficiency alters the proteome of adult rat hippocampal synaptosome. Am J Physiol Regul Integr Comp Physiol. 2013;305: R1297-306.

25. Insel BJ, Schaefer CA, McKeague IW, Susser ES, Brown AS. Maternal iron deficiency and the risk of schizophrenia in offspring. Arch Gen Psychiatry. 2008;65(10):1136-44.

26. Tran PV, Fretham SJB, Carlson ES, Georgieff MK. Long-term reduction of hippocampal BDNF activity following fetal-neonatal iron deficiency in adult rats. Pediatr Res. 2009;65(5): 493-8.

27. - Callahan LSN, Thibert KA, Wobken JD, Georgieff MK. Early life iron deficiency anemia alters the development and long-term expression of parvalbumin and perineuronal nets in the rat hippocampus. Dev Neurosci. 2013;35(5):427-236. This empirical paper documents decreased parvalbumin mRNA expression and protein levels in rodents who had experienced early life ID compared to iron sufficient controls, which is consistent with evidence of delayed hippocampal maturation and lower plasticity in adults who were formerly iron deficient.

28. Hensch TK. Critical period regulation. Annu Rev Neurosci. 2004;27:549-79.

29. Hubel DH, Wiesel TN. Receptive fields, binocular interaction and functional architecture in the cat's visual cortex. J Physiol. 1962;160:106-54.

30. Gluckman PD, Hanson MA. Living with the past: evolution, development, and patterns of disease. Science. 2004;305(5691): 1733-6.

31. - Blegen MB, Kennedy BC, Thibert KA, Gewirtz JC, Tran PV, Georgieff MK. Multigenerational effects of fetal-neonatal iron deficiency on hippocampal BDNF signaling. Physiol Rep. 2013;1(5):1-10. This paper documents acute and chronic BDNF downregulation in formerly iron deficient $F 1$ rats. However, iron sufficient $F 2$ generation rats showed gene expression comparable to controls, suggesting that the effects of ID on BDNF expression are not multi-generational.

32. Roth TL, Sweatt JD. Epigenetic marking of the BDNF gene by early-life adverse experiences. Horm Behav. 2011;59(3):315-20.

33. Felt BT, Beard JL, Schallert T. Persistent neurochemical and behavioral abnormalities in adulthood despite early iron supplementation for perinatal iron deficiency anemia in rats. Behav Brain Res. 2006;171:261-70.

34. Schmidt AT, Alvarez GC, Grove WM, Rao R, Georgieff MK. Early iron deficiency enhances stimulus-response learning of adult rats in the context of competing spatial information. Dev Cog Neurosci. 2012;2(1):174-80.

35. Lozoff B, Beard J, Connor J, Felt B, Georgieff M, Schallert T. Long-lasting neural and behavioral effects of early iron deficiency in infancy. Nutr Rev. 2006;64:S34-43.

36. Morse A, Beard JL, Jones B. Behavioral and neurochemical alterations in iron deficient mice. Proc Soc Exp Biol Med. 1999;220:147-52.

37. Armony-Sivan R, Eidelman AI, Lanir A, et al. Iron status and neurobehavioral development of premature infants. J Perinatal. 2004;24:757-62.

38. Tamura T, Goldenberg RL, Hou J, et al. Cord serum ferritin concentrations and mental and psychomotor development of children at five years of age. J Pediatr. 2002;140(2):165-70.

39. DeBoer T, Wewerka S, Bauer PJ, Georgieff MK, Nelson CA. Explicit memory performance in infants of diabetic mothers at 1 year of age. Dev Med Child Neurol. 2005;47:525-31.

40. Ornoy A. Growth and neurodevelopmental outcome of children born to mothers with pregestational and gestational diabetes. Pediatr Endocrinol Rev. 2005;3(2):104-13.

41. Riggins T, Miller NC, Bauer PB, Georgieff MK, Nelson CA. Consequences of low neonatal iron status due to maternal diabetes mellitus on explicit memory performance in childhood. Dev Neuropsychol. 2009;34:762-79.

42. Angulo-Barroso RM, Schapiro L, Liang W, et al. Motor development in 9-month-old infants in relation to cultural differences and iron status. Dev Psychobiol. 2011;53:196-210.

43. Peirano PD, Algarín CR, Chamorro RA, et al. Sleep alterations and iron deficiency anemia in infancy. Sleep Med. 2010;11(7):637-42.

44. •- Lozoff B, Smith JB, Kaciroti N, Clark KM, Guevara S, Jimenez E. Functional significance of early-life iron deficiency: outcomes at 25 years. J Pediatr. 2013;163:1260-1266. Lozoff and colleagues are the first to demonstrate effects of early-life iron deficiency at 25 years of age. Formerly iron deficient adults were more likely to have social and emotional problems, and they were less likely to complete secondary school, which was mediated by poorer cognitive functioning in adolescence.

45. Algarín C, Nelson CA, Peirano P, Westerlund A, Reyes S, Lozoff B. Iron-deficiency anemia in infancy and poorer cognitive inhibitory control at age 10 years. Dev Med Child Neurol. 2013;55(5):453-8.

46. Christian P, Murray-Kolb LE, Khatry SK, et al. Prenatal micronutrient supplementation and intellectual and motor function in early school-aged children in Nepal. JAMA. 2010;304(24): 2716-23.

47. Christian P, Morgan ME, Murray-Kolb L, et al. Preschool ironfolic acid and zinc supplementation in children exposed to ironfolic acid in utero confers no added cognitive benefit in early school-age. J Nutr. 2011;141(11):2042-8.

48. Cusick SE, Georgieff MK. Nutrient supplementation and neurodevelopment: timing is the key. Arch Ped Adolesc Med. 2012;155:481-2.

49. •- Wachs TD, Georgieff M, Cusick S, McEwen B. Issues in the timing of integrated early interventions: contributions from nutrition, neuroscience and psychological research. Ann NY Acad Sci. 2014;1308:89-106. This paper is a review of the nutritional, neuroscientific, and psychological evidence for sensitive periods of development. Knowledge of these sensitive periods of development for different types of outcomes should inform interventions for children.

50. Cogswell ME, Parvanta I, Ickes L, Yip R, Brittenham. Iron supplementation during pregnancy, anemia, and birth weight: a randomized controlled trial. Am J Clin Nutr. 2003;78:773-81.

51. Lozoff B, De Andraca I, Castillo M, Smith JB, Walter T, Pino P. Behavioral and developmental effects of preventing iron- 
deficiency anemia in healthy full-term infants. Pediatrics. 2003;112(4):846-54

52. Black MM, Baqui AH, Zaman $\mathrm{K}$, et al. Iron and zinc supplementation promote motor development and exploratory behavior among Bangladeshi infants. Am J Clin Nutr. 2004;80(4): 903-10.

53. Stoltzfus RJ, Kvalsvig JD, Chwaya HM, et al. Effects of iron supplementation and anthelmintic treatment on motor and language development of preschool children in Zanzibar: double blind, placebo controlled study. BMJ. 2001;323(7326):1389-93.

54. Grantham-McGregor S, Ani C. A review of studies on the effect of iron deficiency on cognitive development in children. J Nutr. 2001;131:(2S-2):649S-666S; discussion 666S-668S.

55. Corapci F, Radan AE, Lozoff B. Iron deficiency in infancy and mother-child interaction at 5 years. J Behav Dev Pediatr. 2006;27:371-8.

56. Murray-Kolb LE, Khatry SK, Katz J, et al. Preschool micronutrient supplementation effects on intellectual and motor function in school-aged Nepalese children. Arch Pediatr Adolesc Med. 2012;166(5):404-10.

57. • Berglund SK, Westrup B, Hagglof B, Hernell O, Domellof M. Effects of iron supplementation of LBW infants on cognition and behavior at 3 years. Pediatrics. 2013;131:47-55. This iron supplementation intervention for marginally low birth weight infants indicates no improvements in cognitive function at 3 years but a significant improvement in behavior problems.

58. Steinmacher J, Pohlandt F, Bode H, Sander S, Kron M, Franz AR. Randomized trial of early versus late enteral iron supplementation in infants with a birth weight of less than 1301 grams: neurocognitive development at 5.3 years' corrected age. Pediatrics. 2007;120(3):538-46.

59. Fuglestad AJ, Georgieff MK, Iverson SL, et al. Iron deficiency after arrival is associated with general cognitive and behavioral impairment in post-institutionalized children adopted from Eastern Europe. Matern Child Health J. 2013;17:1080-7.

60. Doom JR, Gunnar MR, Georgieff MK, et al. Beyond stimulus deprivation: Iron deficiency and cognitive deficits in post-institutionalized children. Child Dev. 2014.

61. Lozoff B, Clark KM, Jing Y, Armony-Sivan R, Angelilli ML, Jacobson SW. Dose-response relationships between iron deficiency with or without anemia and infant social-emotional behavior. J Pediatr. 2008;152(5):696-702. 